\title{
Impact of Transmission Performances on Path Routing in All-Optical Transport Networks
}

\author{
M. Berdusco ${ }^{+}$, S. Binetti ${ }^{\#}$, E. Iannone ${ }^{+}$, M. Listanti* ${ }^{*}$ R. Sabella \\ + Fondazione Ugo Bordoni, Rome, Italy \\ * INFOCOM Dpt., Università di Roma "La Sapienza" \\ \# Ericsson Telecomunicazioni, $R \&$ D Division, Rome, Italy
}

\author{
Roberto Sabella \\ e-mail: sabella@RD.tei.ericsson.se \\ tel: 06-72583046 fax: 06-72583940
}

\begin{abstract}
The impact of transmission related issues on the routing strategies for transparent all-optical WDM transport networks is analyzed in this paper. Three different categories of routing algorithms are analyzed: algorithms based on the Wavelength Path (WP) strategy, based on the Virtual Wavelength Path (VWP) strategy and requiring only a limited number of wavelength converters in the network (PVWP: Partial Virtual Wavelength Path). It results that the PVWP allows a saving in network devices with respect to the WP similar those permitted by the VWP also attaining transmission performances near those attained by the WP that are quite better that those attained by the VWP.
\end{abstract}

\section{Keywords}

WDM NETWORKS, TRANSPARENT NETWORKS, OPTICAL PATH ROUTING, WAVELENGTH CONVERSION, TRANSMISSION IN OPTICAL NETWORKS

The original version of this chapter was revised: The copyright line was incorrect. This has been corrected. The Erratum to this chapter is available at DOI: 10.1007/978-0-387-35398-2_19 


\section{Part Four: Transmission in optical transport networks}

\section{INTRODUCTION}

Optical transparent WDM networks seems the most promising candidates to constitute the future high-capacity-long distance communication infrastructure. In such networks the routing function is carried out directly in the optical domain by Optical Cross-Connects (OXC) so that high speed optical signals travels through the network without any optical-to-electrical conversion from their source to their destination. On this ground the concept of Optical Path (OP) can be defined, similarly to the concept of electrical path in SDH networks: an OP is a semi-permanent connection between path end points that is set-up utilizing OXCs [1,2].

Three different strategies have been proposed so far for the routing of optical paths in an optical WDM network: the Wavelength Path (WP) [1], the Virtual Wavelength Path (VWP) [1] and the Partial Virtual Wavelength Path (PVWP) [3,4]. In the WP, a fixed wavelength is assigned to each optical path and it is never changed in all its route through the network; in the VWP the wavelengths are assigned link-by-link to each path, so that the path wavelength can be changed at each OXC crossing; in the PVWP only a few OXCs in the network are able to change the wavelength of the crossing paths so that the path wavelength generally remain unchanged at the OXC crossing but for a limited number of changes at specific locations.

These classes of algorithms have been compared both considering the number of required WDM channels per fiber in the case in which a single fiber link exists between each couple of OXCs [5] and considering the required OXC dimension (number of i/o ports) in the case in which the maximum number of wavelengths per fiber is fixed [6,4]. The most significant comparison is the latter, since the number of wavelengths is generally constrained by the adopted technology; its main result is that the VWP and the PVWP allow a sensible save in OXC ports with respect to the WP. Moreover the PVWP reaches this task by using a limited number of wavelength converter devices, that is generally quite smaller that those required by the VWP.

However, transmission issues have never been considered while analyzing routing algorithms. While in an SDH network the transmitted signal is regenerated at each node, no regeneration is present in a transparent WDM network. Therefore noise and distortion accumulates along all the signal route. Since thousands kilometers long paths can be well present in a WDM network with a large geographical extension, perhaps not all the possible routes between a couple of path-end OXCs can be traveled with acceptable transmission performances. Thus, one more constraint is needed in the routing algorithms: all the routed paths must have acceptable transmission performances.

A comparison between WP, VWP and PVWP is for the first time presented in this paper. Specifically, a particular geographical network and a particular OXC technology are considered and different traffic volumes are routed through the network by using WP, VWP and PVWP algorithms. After the routing, each routed path is analyzed under the transmission 
point of view and the Unacceptable Paths Number (UPN: those path that do not assure acceptable transmission performances) is observed.

\section{ROUTING ALGORITHMS}

The routing algorithms used for the WP and the VWP scheme are those reported in [6]. These are based on an heuristic procedure of path accommodation and allows the OXC scale needed to accommodate a fixed traffic volume to be determined. As far as the PVWP is concerned, the so called global arrangement algorithm is used, that is described in [4].

All the routing algorithms accept as input a traffic pattern to accommodate; a traffic pattern is a set of path to be routed and it is represented as a matrix indicating how many paths have to be set-up between each pair of network nodes. A traffic volume, defined as the number of accommodated paths, is associated to each traffic pattern. In this paper we report average results with respect to the traffic pattern; that is we randomly generate a great number of traffic patterns that corresponds to the same traffic volume and average the considered figure of merit with respect to the traffic pattern.

Different figures of merit can be defined to compare routing algorithms. The first figure of merit is the average number of OXC ports $\mathrm{N}$, that gives a measure of the needed OXC scale. Another important figure of merit is related to the number of wavelength converters used in the network: this is the "Wavelength Conversion Percentage Amount" (WCPA), defined as: $\mathrm{WCPA}=\overline{\mathrm{K}} /\left(\mathrm{N}_{1} \mathrm{M}\right)$,

where $\overline{\mathrm{K}}$ is the average number of converters in the OXCs and $\mathrm{M}$ is the number of WDM channels per fiber. The figure of merit we mainly consider in this paper is the average number of paths that do not have acceptable transmission performances (UPN: Unacceptable Paths Number).

\section{OXC ARCHITECTURE AND RELATED TECHNOLOGIES}

To be able to consider transmission issues, the OXC architecture and the technology used to realize the adopted optical devices have to be specified.

In this paper we consider a consolidated OXC architecture, that can be also equipped with a limited number of wavelength converters without spoiling its functionality. This architecture, whose functional scheme is reported in figure 1 , is based on the use of space switching optical matrixes, tunable filters and wavelength converters. In particular the routing is carried out by the space switching matrixes and the wavelength converters prevent conflicts between different paths entering the OXC at the same wavelength and directed towards the same OXC output port. Two Erbium-Doped Fiber Amplifiers are present to compensate the internal loss and part of the input link loss if it is the case. 


\section{Part Four: Transmission in optical transport networks}

The space switching matrixes are assumed to be realized with the thermo-optical technology [14], with a linear loss of $0.8 / \mathrm{N}$ (being $\mathrm{N}$ the number of input ports) and a crosstalk factor equal to $\chi=410^{-6}$.

The tunable filters are assumed to be double-stage Fabry-Perot filter with a 3-dB bandwidth equal to $100 \mathrm{GHz}$ and a loss of $2 \mathrm{~dB}$.

The EDFAs are assumed to have a maximum output power of $18 \mathrm{dBm}$, a flat bandwidth of 35 $\mathrm{nm}$ and a noise factor $\mathrm{F}_{\mathrm{E}}$ of $4 \mathrm{~dB}$.

Finally the wavelength converters are assume to be based on Four-Wave Mixing in Semiconductor Optical Amplifiers (SOA) $[9,10]$. Since the behavior of the wavelength converters is quite important to determine the network transmission performances, it has to be described quite in detail. The model adopted to describe the performances of the wavelength converters is those reported in [9]. The wavelength converters parameters are relative to a low noise SOA, $1.5 \mathrm{~mm}$ long [15]. It is to be noted that, each time an optical signal experiences wavelength conversion, it is assumed that it is translated from lower limit of the WDM comb to the higher one, so to perform a worst case performance evaluation.

\section{TRANSMISSION EVALUTATION}

In order to evaluate the transmission performances of the optical path we assume that in-line optical amplification is used in the network, with an amplifier spacing $\mathrm{L}_{\mathrm{A}}=50 \mathrm{~km}$. The amplifier gain exactly compensates for the loss of the fiber link before the amplifier and the EDFAs inside the OXC compensate both the OXC internal losses and the loss of the fiber link between the last amplifier and the OXC. The fibers in the network are assumed to be Dispersion Compensating fibers with a dispersion $\mathrm{D}=4 \mathrm{ps} / \mathrm{nm} / \mathrm{km}$ and an attenuation $\alpha=0.25$ $\mathrm{dB} / \mathrm{km}$.

We assume that nonlinear fiber propagation can be neglected in our network. This means that the transmitted optical power has to be quite lower than the threshold power for the Brillouin and Raman effects and that the characteristic dispersion length $L_{D}$ is always much smaller that the nonlinear length $\mathrm{L}_{\mathrm{NL}}$ during signal propagation [16] so that even Kerr effect can be neglected.

Under these conditions, the main phenomena affecting the transmission performances of the optical paths are the ASE accumulation along the link and the in-band crosstalk inside the OXCs [15].

In this regime, the $\mathrm{Q}$ factor can be assumed as a good figure of merit to characterize the transmission performances of the optical paths. The $\mathrm{Q}$ factor is defined as the electrical signal-to-noise ratio after the baseband filter at the receiver; in the Gaussian approximation (that holds fairly well in our case) an error probability of $10^{-9}$ corresponds to an $\mathrm{Q}$ factor equal 
to 6. To assume a reasonable system margin, we will classify as unacceptable under the transmission point of view all the optical path for which the $Q$ factor is lower than 6.5.

In our case, the $Q$ factor has the following expression

$$
\mathrm{Q}=\frac{\mathrm{SNR}_{\mathrm{o}}}{\sqrt{2 \mathrm{SNR}_{\mathrm{o}} \mathrm{b}}+\sqrt{\mathrm{b}}} .
$$

where SNRo is the optical signal to noise ratio in the signal bandwidth and $b$ the ratio between the overall optical bandwidth and the electrical bandwidth (determined by the baseband filter after the receiver). We assume that the single channel bit-rate is equal to 2.5 $\mathrm{Gbit} / \mathrm{s}$ and that $\mathrm{b}=5$, condition that can be realized using an optical pre-detection filter with a bandwidth of $25 \mathrm{GHz}$. The channel spacing $\Delta v$ is set to $300 \mathrm{GHz}$.

The Optical signal to noise ratio can be written as

$\mathrm{SNR}_{\mathrm{o}}=\frac{\rho \mathrm{P}_{\mathrm{r}}}{2 \mathrm{RS}_{\mathrm{tot}}}$.

where $\rho$ is the penalty due to the in-band crosstalk, $P_{r}$ and $S_{\text {tot }}$ are the peak optical power and the overall ASE power spectral density impinging the receiver.

The received optical power is simply proportional to the power at the output of each OXC and each EDFA by a factor depending on the loss of the path from the last OXC input to the receiver.

The penalty $\rho$ can be evaluated by using the equivalent Gaussian noise approximation, as shown in [17]. In particular, $\rho$ is a function of the overall crosstalk-to-signal ratio (CSR) whose expression in the worst case (wavelength translated signal against an unchanged crosstalk component) is simply given by

$\mathrm{CSR}=\sum_{\mathrm{k}=1}^{\mathrm{NN}}\left[\frac{|\mathrm{H}(\Delta v)|^{2}}{\eta_{\mathrm{k}}}+\chi \mathrm{M}\right]$.

where $\mathrm{NN}$ is the number of traveled OXCs, $\mathrm{H}(v)$ is the frequency response of the tunable filters inside the OXC, $\Delta v$ is the channel spacing, $\chi$ is the crosstalk factor of the space switching matrixes and $M$ is the number of WDM channels per fiber. The factor $\eta_{k}$ is equal to the wavelength converter efficiency if the considered path experiences wavelength conversion inside the $\mathrm{k}$-th $\mathrm{OXC}$, otherwise it is equal to one.

Finally the overall ASE power spectral density can be evaluated starting from the physical parameters of the different optical devices as detailed in [18]. The term $S_{\text {tot }}$ depends heavily on the way in which the overall gain inside the OXC is divided between the two available EDFAs. In particular, minimizing $S_{\text {tot }}$, it can be demonstrated that the lowest noise power is obtained when the gain of the first amplifier is maximized. Thus, the amplifier at the OXC input has the maximum gain, that is determined by two constraints: first of all the maximum 


\section{Part Four: Transmission in optical transport networks}

optical power the amplifier can emit in the signal bandwidth is limited (18 dBm in our case), second, the signal cannot be amplified so much that a possible output wavelength converter exists from a pump-probe working regime. This requires that, if $P_{p}$ is the wavelength converter pump power, the signal power at the wavelength converter input is at least $10 \mathrm{~dB}$ smaller than $\mathrm{P}_{\mathrm{p}}$, thus limiting the gain of the input EDFA.

\section{SIMULATION RESULTS}

In order to provide simulation results, we consider in this paper a slightly modified version of the high speed Italian transport network. The network topology is shown in figure 2.

No optimization of the signal power and of the pump power of the wavelength converters is attempted in this paper. The signal power at the output of each OXC is fixed to $2 \mathrm{dBm}$, a sufficiently low value to neglect nonlinear effects during fiber propagation. The pump power of the wavelength converters is fixed to $8 \mathrm{dBm}$. Since this is the power injected into the SOA, this generally requires a pump laser emitting at least $14 \mathrm{dBm}$.

The average number of OXC ports versus the traffic volume is shown in figure 3 for the WP and the VWP algorithms. The results relative to the PVWP algorithm coincides with those relative to the VWP. The average number of ports increases at the increasing of the traffic volume and decreases at the increasing of the number of channels per fiber. Moreover, the little gain due to the adoption of wavelength conversion is evident from figure 3.

The average Wavelength Conversion Percentage Amount (WCPA) needed to implement the PVWP algorithm is shown in figure 4 versus the traffic volume. From the figure it results that the number of wavelength converters really needed to attain the performances of the VWP is quite smaller than $100 \%$. Moreover the WCPA decreases at the increasing of the traffic volume. This is due to the fact that, increasing the traffic volume, the average number of OXC ports increases so that the routing map given by the WP is more and more similar that given by the VWP. This means that the number of needed wavelength conversions tends to decrease, as shown in figure 4.

The average Unacceptable Path Number (UPN) is shown in figure 5 versus the Normalized Traffic Volume (NTV), the traffic volume divided by the number of nodes, for the VWP algorithm and for $M=4,6$. In the case $M=8, U P N=100 \%$ for all the considered traffic volumes. Before discussing these results, an observation is needed. When we evaluate the transmission performances of an optical path including a certain number of wavelength converters, we assume that wavelength conversion really occurs at every converters with the maximum conversion range, i.e. with the minimum efficiency. This is a worst case approach that is far from reality for a large number of paths in the case of the VWP. If this approximation is removed and optimization of the transmitted power and of the pump power 
of the wavelength converters is carried out, better transmission performances can be obtained $[19,20]$.

From figure 5, it can be observed that UPN increases at the increasing of the NTV. This is due to the fact that the average number of ports enlarges, thus increasing the OXC internal loss. Moreover, UPN rapidly growths with M, despite the decreasing of the average number of ports. This is due to two factors: the overall internal losses of the OXC increases and the efficiency of the wavelength converters decreases due to the greater conversion range. As far as the internal loss is concerned, it is the product of the losses of the input splitter, the filter, the SSM and the output combiner. The filter loss does not depend on M, the product of the other losses, assuming that the overall $\mathrm{OXC}$ capacity $\mathrm{C}=\mathrm{M} \mathrm{N}$ remains constant, depends on $1 / \mathrm{M}$. Concerning the efficiency, in our case, for $M=4$ it is about $-6.8 \mathrm{~dB}$, for $M=6$ it is -11.3 $\mathrm{dB}$ and for $\mathrm{M}=8$ it is $-14.7 \mathrm{~dB}$.

The dependence of UPN on NTV in the case of the PVWP algorithm is shown in figure 6. The curves for $\mathrm{M}=4$ is not reported in the figure since $\mathrm{UPN}=0$ in all the considered cases. Since the number of wavelength converters in the network is by far smaller than in the case of the VWP, even UPN is quite smaller. With M=6 UPN is smaller than $2 \%$ up to NTV $=150$.

\section{Conclusions}

Transmission problems cannot be neglected in designing routing algorithms for wide area transparent WDM networks. In this paper, the impact of the main transmission issues on the path routing is analyzed in the case of a typical wide area network (a modified form of the high capacity Italian network) assuming to route optical paths by Optical Cross-Connects based on space switching and allowing, if the case, even wavelength conversion. Wavelength converters based on Four-Wave Mixing in Semiconductor amplifiers are considered. While in the case of routing based on the Wavelength Path scheme, the transmission issues are not important, they have to be considered in the case of the adoption of the Virtual Wavelength Path or of the Partial Virtual Wavelength Path schemes. In this cases, it can occur that some optical path do not have acceptable transmission performances. Two ways are possible to cope with this problem: the unacceptable paths can be routed again on a different route presenting good transmission performances by further enlarging the OXC scale or electronic regenerators can be put on the unacceptable path, so spoiling the network optical transparency.

Comparing the VWP and the PVWP, it results that the number of unacceptable paths is quite larger in the case of the VWP, due to the larger number of wavelength converters crossed by each path. This is another important advantage of the PVWP, besides the possibility to attain the same routing performances of the VWP with simpler OXCs. 


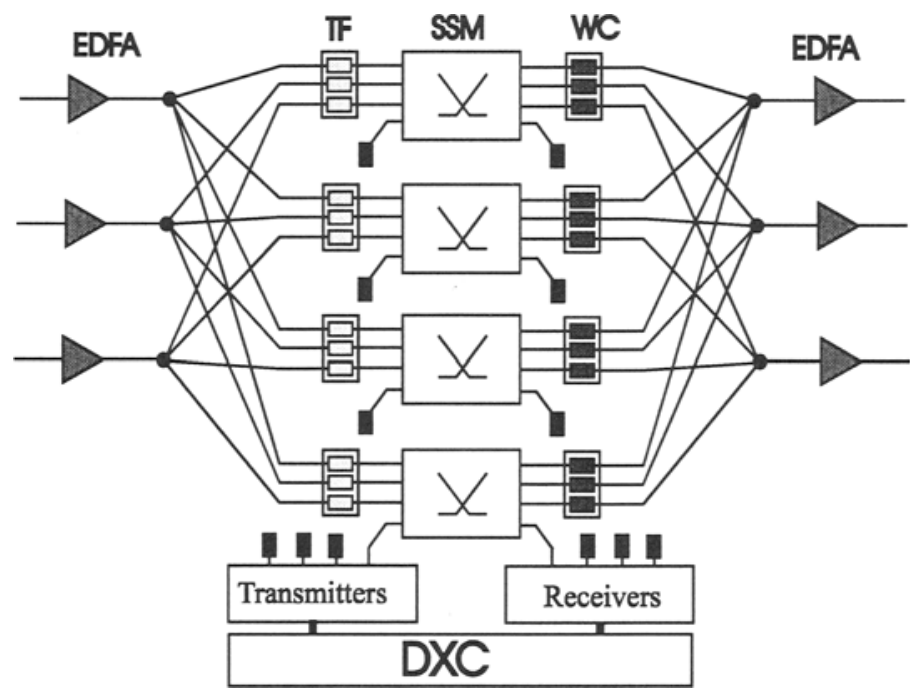

Figure 1 OXC Architecture

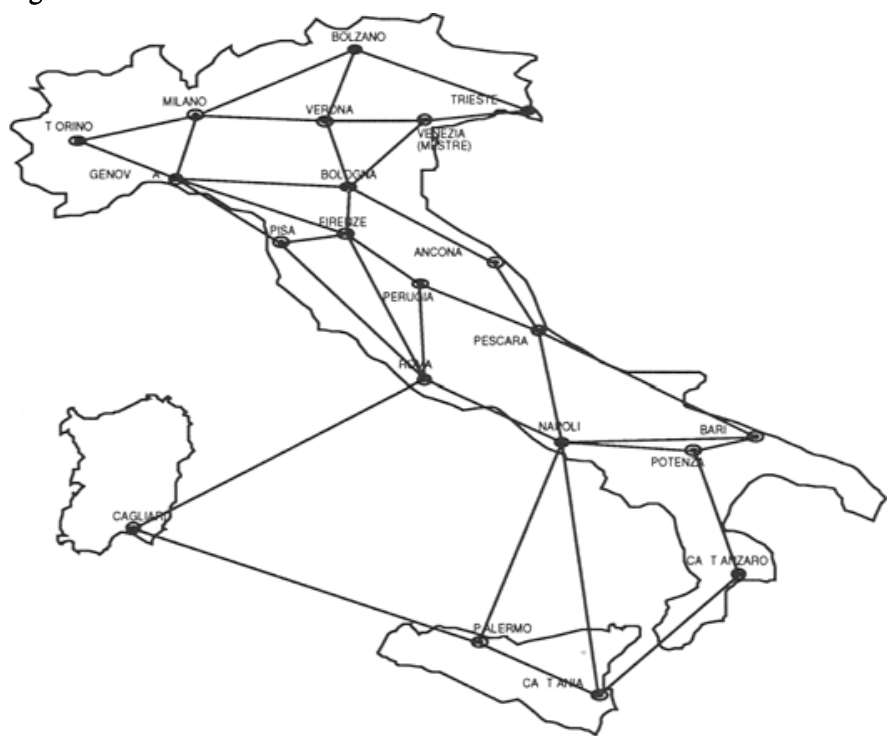

Figure 2 Topology of the high-capacity Italian network used in this paper. 


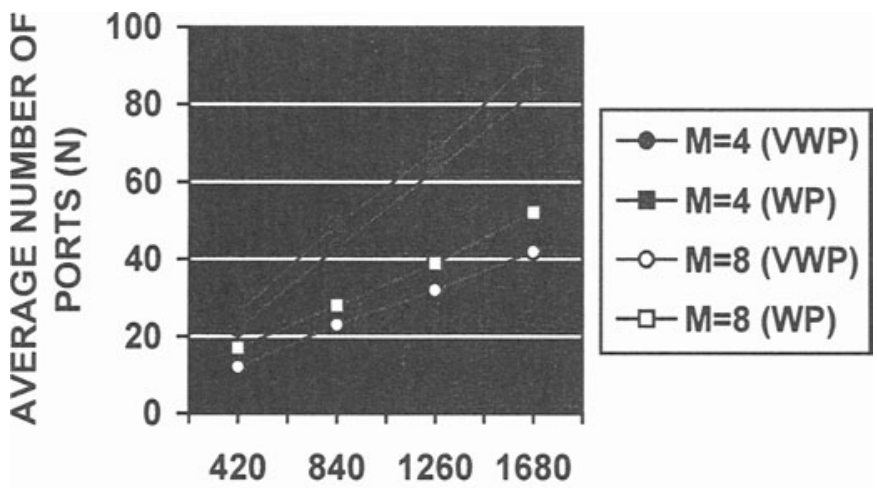

\section{TRAFFIC VOLUME}

Figure 3 Average number of ports versus the traffic volume for the VWP algorithms.

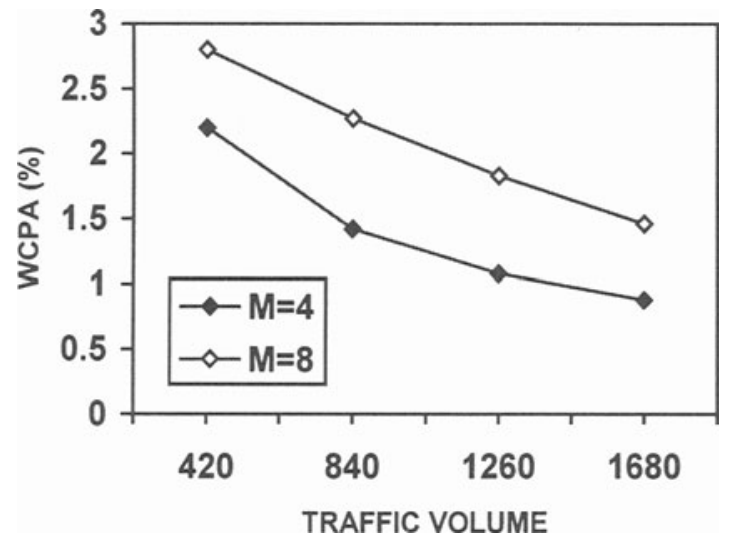

Figure 4:Average Wavelength Conversion Percentage Amount (WCPA) versus the traffic volume. 


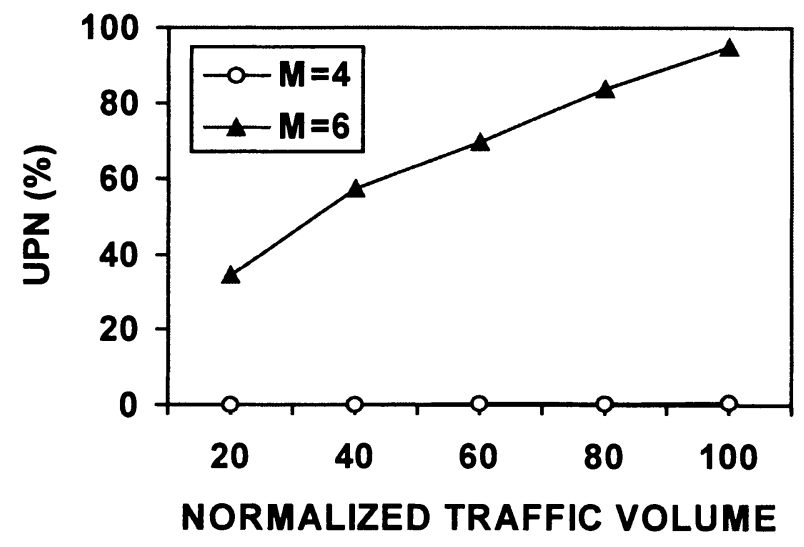

Figure 5:Average Unacceptable Path Number (UPN) versus the Normalized Traffic Volume for the VWP algorithm.

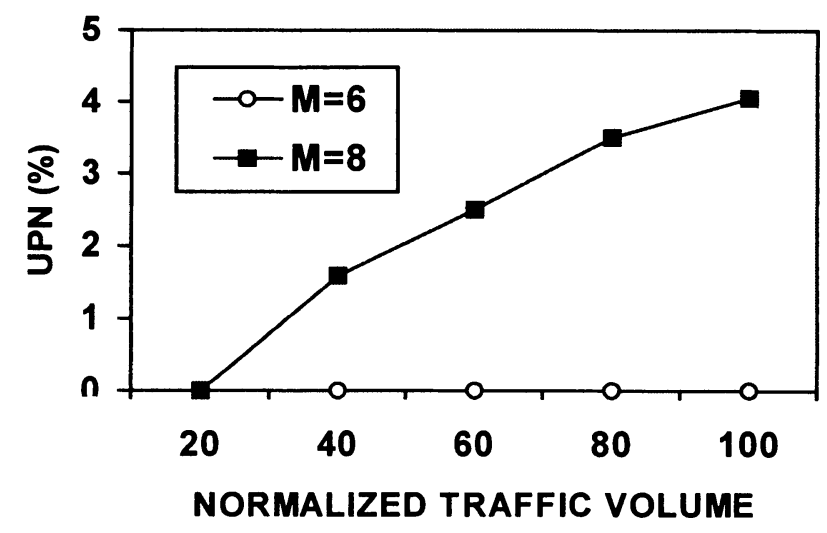

Figure 6:Average Unacceptable Path Number (UPN) versus the Normalized Traffic Volume for the PVWP algorithm. 


\section{REFERENCES}

[1] K. Sato, S. Okamoto and H.Hadama, "Network performance and integrity enhancement with optical path layer technologies", IEEE-Journal on Selected Areas in Communications, vol. 12, no. 1, pp. 159-170, 1994.

[2] R. Ramaswami, K.N. Sivarajan, "Routing and Wavelength Assignment in All-Optical Networks", IEEE/ACM Transaction on Networking, vol. 3, no. 5, pp. 489-500, 1995.

[3] M. Listanti, M. Berdusco, R. Sabella, "Optical Path Strategies in WDM All-Optical Networks: Minimization of Wavelength Converters in Optical Cross-Connect", GLOBECOM '97, November 1997, Phoenix, AZ.

[4] M. Listanti, M. Berdusco, R. Sabella, "A New Strategy For Employing Wavelength Conversion in WDM Optical Networks”, LEOS '97, November 1997, San Francisco.

[5] N. Nagatsu, Y. Hamazumi, K. Sato, "Optical Path Accommodation Designs Applicable to Large Scale Networks”, IEICE Transactions on Communications, vol. E78-B, n 4, pp. 597-607, 1995.

[6] N. Nagatsu, et al., "Optical Path Cross-Connect System Scale Evaluation Using Path Accommodation Design For Restricted Wavelength Multiplexing", IEEE Journal of Selected Areas on Communications, vol. 14, no. 5, pp.893-902, 1996.

[7] A. Watanabe, S. Okamoto, K. Sato, "Optical path cross-connect node architecture with high modularity for photonic transport networks", IEICE Transaction on Communications, vol. E77-B, no. 10, pp. 1220-1229, 1994.

[8] G.R. Hill et al., "A transport network layer based on optical network elements", IEEE-Journal of Lightwave Technol., vol.11, no.5/6, pp. 667-679, 1993.

[9] A. D'Ottavi, E. Iannone, A. Mecozzi, S. Scotti, P. Spano, R. Dall'Ara, J. Eckner, G. Guekos,"Efficiency and Noise Performances of Wavelength Converters Based on FWM in Semiconductor Optical Amplifiers", IEEE-Photonics Technology Letters, vol. 31, no. 4, 1995.

[10] R. Ludwig, G. Raybon, "BER Measurements of Frequency Converted Signals using Four-Wave Mixing in a Semiconductor Laser Amplifier at 1, 2.5, 5 and $10 \mathrm{Gbit} / \mathrm{s}$ "; Electronics Letters, vol. 30, n 4, pp. 338-339, 1994. 
96 Part Four: Transmission in optical transport networks

[11] S. J. B. Yoo, C. Caneau, R. Bhat, M. A. Koza, A. Rayhil, N. Antoniades, "All-Optical Wavelength Conversion by Quasi-Phasematched Difference Frequency Generation in AlGaAs Waveguides”; Applyed Physics Letters, vol. 68, pp. 2609-2610, 1966.

[12] M. Bischoff, M. N. Huber, O. Jahreis, F. Derr, F. Hulm, "Operation and Maintainance for an AllOptical Transport Network”; IEEE-Communication Magazine, vol. 11, pp. 136- 142, 1996.

[13] T. Durhuus, B. Mikkelsen, C. Joergensen, S. L. Danielsen, K. E. Stubkjaer, "All-Optical Wavelength Conversion by Semiconductor Optical Amplifiers",.IEEE-Journal of Lightwave Technology, vol. 14, n 8, pp. 942-954, 1996.

[14] M. Koga, A. Watanabe, S. Okamoto, K. Sato, M. Okuno, "8x16 delivery-and-coupling-type optical switches for a 320-Gb/s throughput optical path cross-connect system", OFC '96 Technical Digest, Paper ThN3, 1996.

[15] E. Iannone, R. Sabella, L. de Stefano, F. Valeri, "All-optical wavelength conversion in optical multicarrier networks", IEEE Transactions on Communications, vol. 44, no. 6, pp. 716 - 724, 1996.

[16] G. P. Agrawal, Nonlinear Fibre Optics, Academic Press, New York, 1989.

[17] L. Moura, N. Karafolas, P. Lane, A. Hill, J. O'Reilly, "Modeling of Interferometric Crosstalk in Optical Networks”; Proceedings of GLOBECOM '96, London, November 1996, pp. 333-337.

[18] E. Iannone, R. Sabella, "Performance Evaluation of an Optical Multi-Carrier Network using wavelength Converters based on FWM in Semiconductor Optical Amplifiers", IEEE-Journal of Lightwave Technology, vol. 13, no. 2, pp. 312-324, 1995.

[19] R. Sabella, E. Iannone, E. Pagano, "Optical Transport Networks Employing All-Optical Wavelength Conversion: Limits and Features", IEEE Journal of Selected Areas in Communications, vol. 14, no. 6, 1996.

[20] E. Iannone, R. Sabella, L. de Stefano, F. Valeri, "All-optical wavelength conversion in optical multicarrier networks", IEEE Transactions on Communications, vol. 44, no. 6, pp. 716 - 724, 1996. 